\title{
Wireless Charging Pad Detection and Alignment Using a Fisheye Camera for Electric Vehicles
}

\author{
Nauman Javed ${ }^{1}$, David Guerrero ${ }^{1}$, Rupert Young ${ }^{2}$, Phil Birch ${ }^{2}$, Chris Chatwin ${ }^{2}$ \\ ${ }^{1}$ Application Solutions Ltd., \\ Continental Corporation (United Kingdom) \\ ${ }^{2}$ Department of Engineering and Design, \\ University of Sussex, \\ Brighton, United Kingdom, BN1 9QT \\ E-mail: $\underline{\text { r.c.d.young@sussex.ac.uk }}$
}

\begin{abstract}
The market for electric vehicles is growing day by day and electric car chargers can be seen often on pavements of the major cities and towns. With this growing market, industry is already looking for another breakthrough, i.e. wireless vehicle charging. This is much like charging smart phones using wireless charging pads instead of plugging the vehicle in. Industry is exploring ways to charge Electric vehicles wirelessly when the car is parked over a charger on the ground beneath it. For the wireless charging to work, both elements must be well aligned. This paper explores using vision based approaches to provide the automatic recognition, localisation and tracking of an inductive plate for wireless car charging. Visual feedback is provided to a motion control system for accurate charger alignment.
\end{abstract}

Keywords: Electric vehicles, inductive charging, automated visual guidance, pattern recognition

\section{INTRODUCTION}

Some modern electric vehicles are equipped with a magnetic charging panel underneath them. A wireless charging station is installed in a parking space or a home garage, where a magnetic pad is installed on the ground. The dimensions of the charging pad used in this study are $690 \times 765 \times 50 \mathrm{~mm}$. The pad includes a specific pattern designed to ease the visual detection by automated guidance algorithms. The pattern will be located on top of the pad. Selection of the optimal pattern is discussed in detail in Section 2. Only 50\% of the total area of the pad is available for the target pattern and is centred as shown in Figure 1.

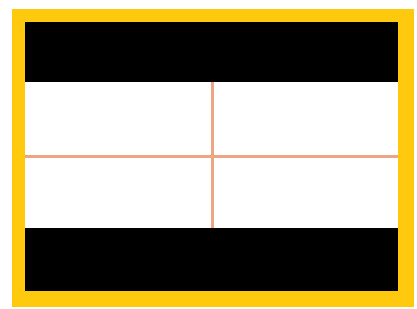

Figure 1. Schematic of charging pad target 
The desired detection distance is $5 \mathrm{~m}$ from the magnetic panel installed in the vehicle. The panel is $1.5 \mathrm{~m}$ behind the frontal camera in the vehicle that is used for this study. Therefore, the pad must be detected at a distance of $3.5 \mathrm{~m}$ from the camera.

Section 3 discusses some algorithms initially considered to solve the problem and Section 4 then describes the methods chosen to first segment the input scene and then accurately align the car to the template pattern. Section 5 summarizes the extensive results obtained to determine the accuracy of the method developed. Section 6 gives a brief conclusion to the work.

\section{OPTIMAL TARGET PATTERN SELECTION}

Target detection ${ }^{2}$ techniques in general are based on natural features that are extracted from the target images and then compared at run time with features in the live camera image. Various target designs were explored to find the optimal target that is trackable and can be accurately detected. The following criteria were used in selection of an appropriate target:

- Good contrast, with both bright and dark regions when well lit

- $\quad$ Rich in detail with collages and a mixture of items

- No repetitive patterns

Another limitation that makes the target detection more challenging in our case is that a fisheye corrected image will be used as input to the algorithm. This limitation is further discussed in Section 4.

Another limitation that was considered while selecting the target pattern was the viewing angle. It is harder to detect and track the target if the camera is looking at the target from a very steep angle, or the target appears very oblique with regards to the camera. The vehicle that was used for this study had the camera mounted at its front and so when the car was some distance from the target pattern the images were acquired at significantly oblique angles.

The table shows the selected target pattern where the $\mathrm{T}$ shape has been chosen as the desired target pattern.

\begin{tabular}{|l|l|l|l|l|}
\hline Pattern & $\begin{array}{l}\text { 3D view } \\
\text { 4m from camera, } \\
40^{\circ} \text { position, } \\
\mathbf{4 0} \text { rotation }\end{array}$ & $\begin{array}{l}\text { Top down view } \\
\text { 4m from camera, } \\
\mathbf{4 0} \text { position, } \\
\mathbf{4 0} \text { rotation }\end{array}$ & Considerations & Conclusion \\
\hline T-shape & - & & $\begin{array}{l}\text { Good frequency } \\
\text { transitions, unique } \\
\text { orientation }\end{array}$ & Accepted \\
\hline & & & & \\
\hline
\end{tabular}

Table 1. Selected Target 


\subsection{Template matching}

\section{AGORITHMS INVESTIGATED}

This method takes an image of the template and the top down view as inputs. Then, it iterates the template matching algorithm to try to find the template in the image, turning each time the template by $1^{\circ}$. It works well but the main drawback is the execution time that is in tens of seconds.

\subsection{FFT based correlation}

This method processes an FFT of the top down image view and template images. The image spectra are then multiplied in the frequency domain. Processing the inverse FFT ${ }^{8}$ of the result allows location of the template's maximum correlation peak. This method has been tested in two different ways:

- $\quad$ To pre-locate the pattern, finding a ROI around the pattern (at steps of $45^{\circ}$ )

- $\quad$ To locate precisely the pattern in an image (at steps of $5^{\circ}$ )

This method is variant to size and rotation. In the top down image, the size of the pattern is known but the rotation is not. Therefore, it is time consuming when used on a large image, such as the top down view image. Therefore, the steps for the pre-location are larger than for the ROI location.

\subsection{Feature descriptors matching}

Methods such as the Harris corner detector ${ }^{7}, \mathrm{SURF}^{4}, \mathrm{SIFT}^{9}, \mathrm{FAST}^{10}$ etc. are the state-of-the-art algorithms for object recognition. For use in the present application, however, unfortunately they are not suitable.

The main problem is that the object is too distorted, and the density of pixels is too low (with the neighbouring regions drastically changed). Shape and intensity of transitory pixel regions fluctuate too much according to the template position. With a template size of $78 * 36$ pixels no SURF points are found on the template image. Even if some Harris points are found, their descriptors do not correlate enough to create a match.

It is possible that adjusting the parameters would improve these preliminary results. Due to a time constraint, however, the approach was discarded in favour of other approaches that seem immediately more promising.
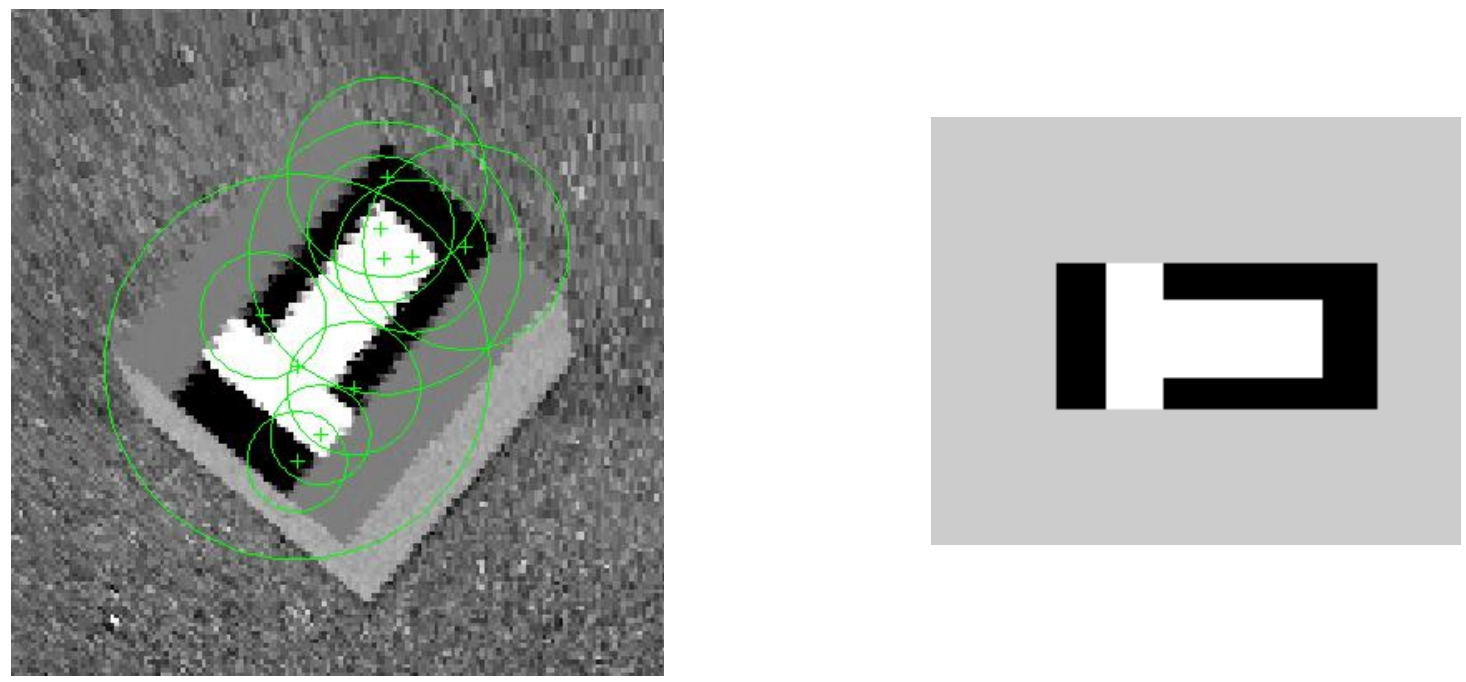
Figure 2

Left - SURF points on top down view

Right - SURF points on the template (none found)
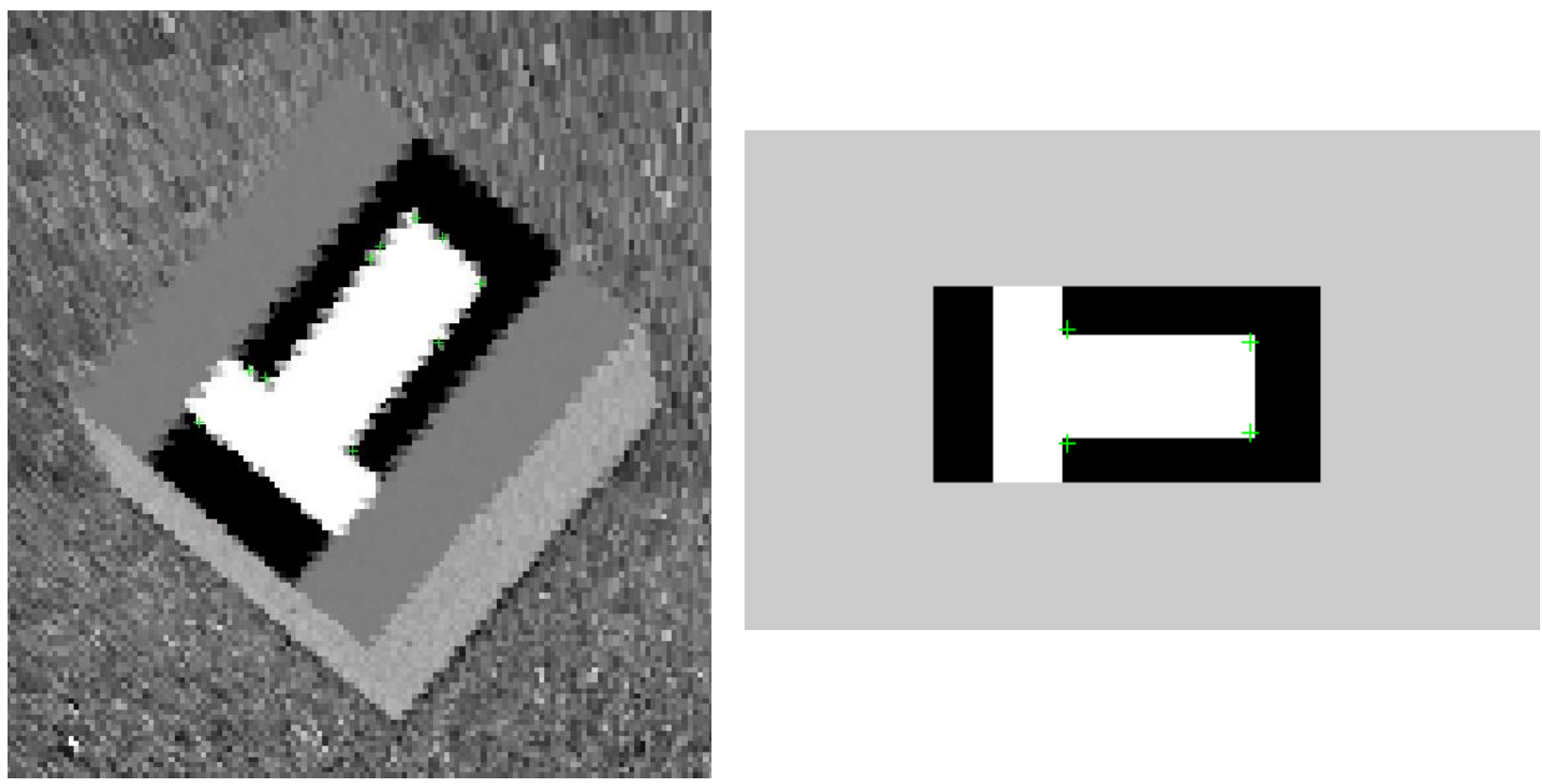

Figure 3

Left: Harris corner points on the top down view (green dots)

Right: Harris corners points on the template (green dots)

\subsection{Custom segmentation}

This approach consists of segmenting the image using known constraints. The segmentation tested tries to isolate white (bright) pixels on the top down image using a thresholding operation. The reasoning behind this is that the pattern is pure white. We then apply a blob algorithm and filter the detected groups by size thresholds. This method does not locate precisely the pattern, but it provides one or multiple ROIs which contain the pattern. Furthermore, this method is not computationally expensive and can be further adapted to take advantage of the prior knowledge of the pattern.

\subsection{Hough Line Detection}

This method aims to detect lines ${ }^{6}$ in images. At first sight, it looks sensible to use this method in order to detect the pattern in the image. After some tests, it was found, however, that this method is disrupted by the noise generated by the top-down view conversion. In the best cases line slices are detected, but not the overall template shape even on a smaller ROIs. Repeatability of this method is thus not reliable.

\subsection{Cross-Correlation}

This method correlates the template and the top down view ${ }^{5}$. The maximum of the resulting image is extracted to locate the pattern ${ }^{11}$. This method is time consuming with large images. Also, it is not invariant to shape and rotation, so it needs 
to be repeated with different rotation angles of the pattern. For these reasons, it is more sensible to use this method on a extracted ROI from the top down view.

Table 2 summarizes the pros and cons for various feature detector approaches explored for this application.

\begin{tabular}{|c|c|c|c|}
\hline Name & Test & Status & Comments \\
\hline Template matching & Discarded pattern at $5 \mathrm{~m}, 0^{\circ}$ & Discarded & $\begin{array}{l}\text { Execution time } \\
\text { much too long }\end{array}$ \\
\hline FFT based convolution & FFT-inverse FFT & On going & $\begin{array}{l}\text { Not independent to } \\
\text { rotation and scale, } \\
\text { still can work in } \\
\text { certain conditions }\end{array}$ \\
\hline Feature Descriptors matching & SURF, HARRIS & Discarded & $\begin{array}{l}\text { Area to be detected } \\
\text { too distorted and not } \\
\text { enough pixels }\end{array}$ \\
\hline Custom segmentation & Binary thresholding + blobs & On going & Useful to find a ROI \\
\hline Hough Line Detection & Standard Hough transform & Discarded & $\begin{array}{l}\text { Not reliable due to } \\
\text { distortion/noise }\end{array}$ \\
\hline Cross Correlation & 2D Cross Correlation & On going & $\begin{array}{l}\text { Interesting in } \\
\text { precision, requires } \\
\text { runtime } \mathrm{o}\left(\mathrm{m}^{2} \mathrm{n}^{2}\right)\end{array}$ \\
\hline
\end{tabular}

Table 2. Summary of various feature detectors

\section{PROPOSED ALGORITHM}

The proposed algorithm involves a two-stage process: a first step which extracts a region of interest; and a second step that computes with more precision the template position and rotation. 


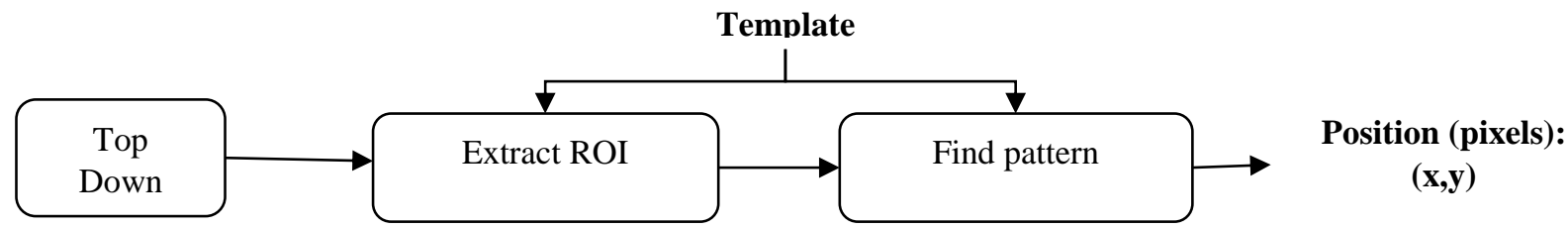

Figure 4. Algorithm Stages

To extract a ROI, two methods have been further explored:

- Custom segmentation

- $\quad$ FFT based correlation

To find the template position and rotation, again two methods have been further explored:

- Normalized Cross Correlation

- $\quad$ FFT based correlation

The algorithm flow pipeline is as shown in Figure 5 below.

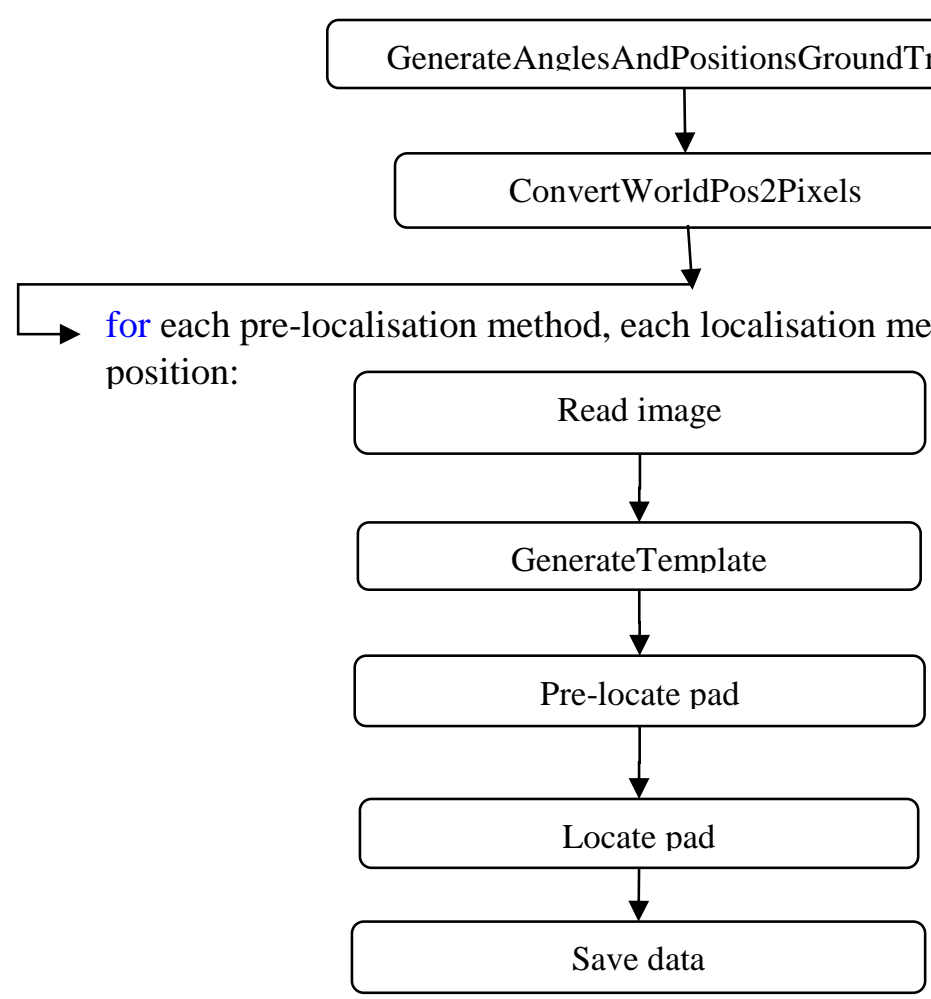

Figure 5. Algorithm Flowchart

The algorithm is broken down into the following stages.

\subsection{Top down view}

Input to the algorithm is a top down view generated using the concept of a virtual view port. A virtual viewport is an imaginary plane placed on the ground at a particular distance and orientation from the camera. As the camera intrinsic 
and extrinsic properties are known, the viewport contains a fisheye corrected view on the ground plane as shown below in Figure 6.

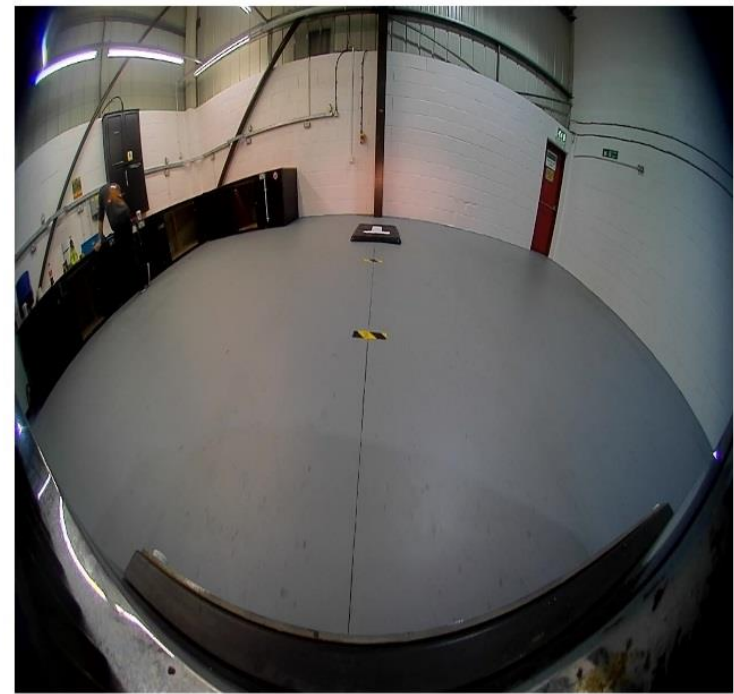

Figure 6 (a) Fisheye Raw View

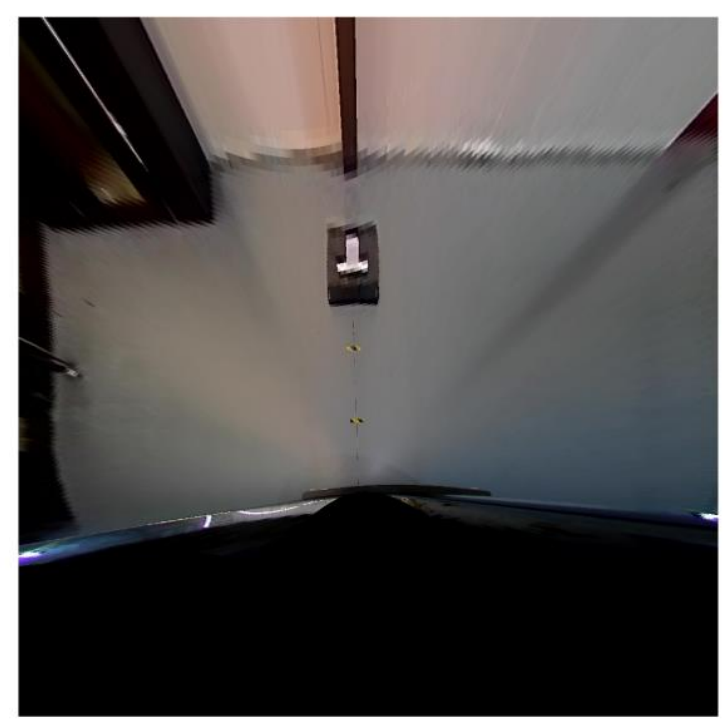

(b) Fisheye Top Down View

\subsection{Pre-locate pad}

Pre-location of the pad calls one of the implemented methods of pre-locating the pad: custom segmentation or FFT based.

\subsubsection{Pre-locate pad, segmentation}

This stage binarizes the top down view image using a threshold of 95\% so only the brightest pixels are segmented. Then, a blob detection algorithm is used to find connected areas, and these are filtered according to their surface. Only those within $35 \%$ of the original template size are selected. Then, a ROI is created centred on the blob together with a margin of 50 pixels.

\subsubsection{Pre-locate pad, FFT Correlation}

Using the whole top down view image and the template image, this function calculates the correlation of both images and gets the maximum value. To take into consideration rotation, the correlation is calculated for a range of angles of the template (between $\left[-180^{\circ}, 180^{\circ}\right]$ at $30^{\circ}$ steps).

The correlation with the maximum value is kept and used to pre-locate the pad. Only correlations resulting in maximum values above a certain threshold are considered to avoid pre-locating non-existing pads.

Binarization, some morphology operations and a blob detection algorithm are performed to create a blob where the peak of the correlation occurs, and the ROI is calculated using its centre and a margin of 50 pixels. 


\subsection{Locate Pad}

This stage calls one of the implemented methods of pre-locating the pad: normalized cross correlation or FFT based correlation.

\subsubsection{Locate pad, FFT based correlation}

Very much in the same fashion as the pre-localisation, this approach calculates the correlation of the template at finer degrees of rotation $\left(5^{\circ}\right.$ steps $)$ with the ROI. The rotation yielding the highest correlation is kept and the position of the pad is given using the coordinates of the maximum value of the correlation result.

\subsubsection{Locate pad, normalized cross-correlation}

2D normalized cross correlation is used for this approach. Again, steps of $5^{\circ}$ are used to cross-correlate the template and the ROI images. The maximum yielding angle is kept, and the result is this angle for the rotation of the pad, the location of the pad being the coordinates of the maximum of the normalized cross-correlation peak.

\section{RESULTS}

From our analysis in Section 4, two methods have been further tested for the pre-localisation and another two for the localisation, giving a total of four combinations:

\begin{tabular}{|l|l|l|l|l|}
\hline & Method 1 & Method 2 & Method 3 & Method 4 \\
\hline Pre-localisation & FFT & Segmentation & Segmentation & FFT \\
\hline Localisation & FFT & FFT & Cross-Correlation & Cross-Correlation \\
\hline
\end{tabular}

To thoroughly test all methods, scenarios were created with the synthetic images in order to place the wireless charging pad to be detected at different distances and angles from the camera.

Due to symmetry, only positions at the left of the camera have been produced. The positions are always at regular distances from the camera and then situated at different angles in steps of $20^{\circ}$. To test the rotation of the pad, different angles have been generated with steps of $15^{\circ}$. These angles have been generated with their centre aligned to the camera and then the possible rotation angles considered are $-90^{\circ}$ and $+90^{\circ}$ of the angle. Some examples of the detection with example top down view images are shown in Figure 7 below.

\begin{tabular}{|l|l|}
\hline $3.5 \mathrm{~m}, 20^{\circ}$ from camera, $5^{\circ}$ pad's rotation & $3.5 \mathrm{~m}, 8^{\circ}$ from camera, $125^{\circ}$ pad's rotation \\
\hline
\end{tabular}




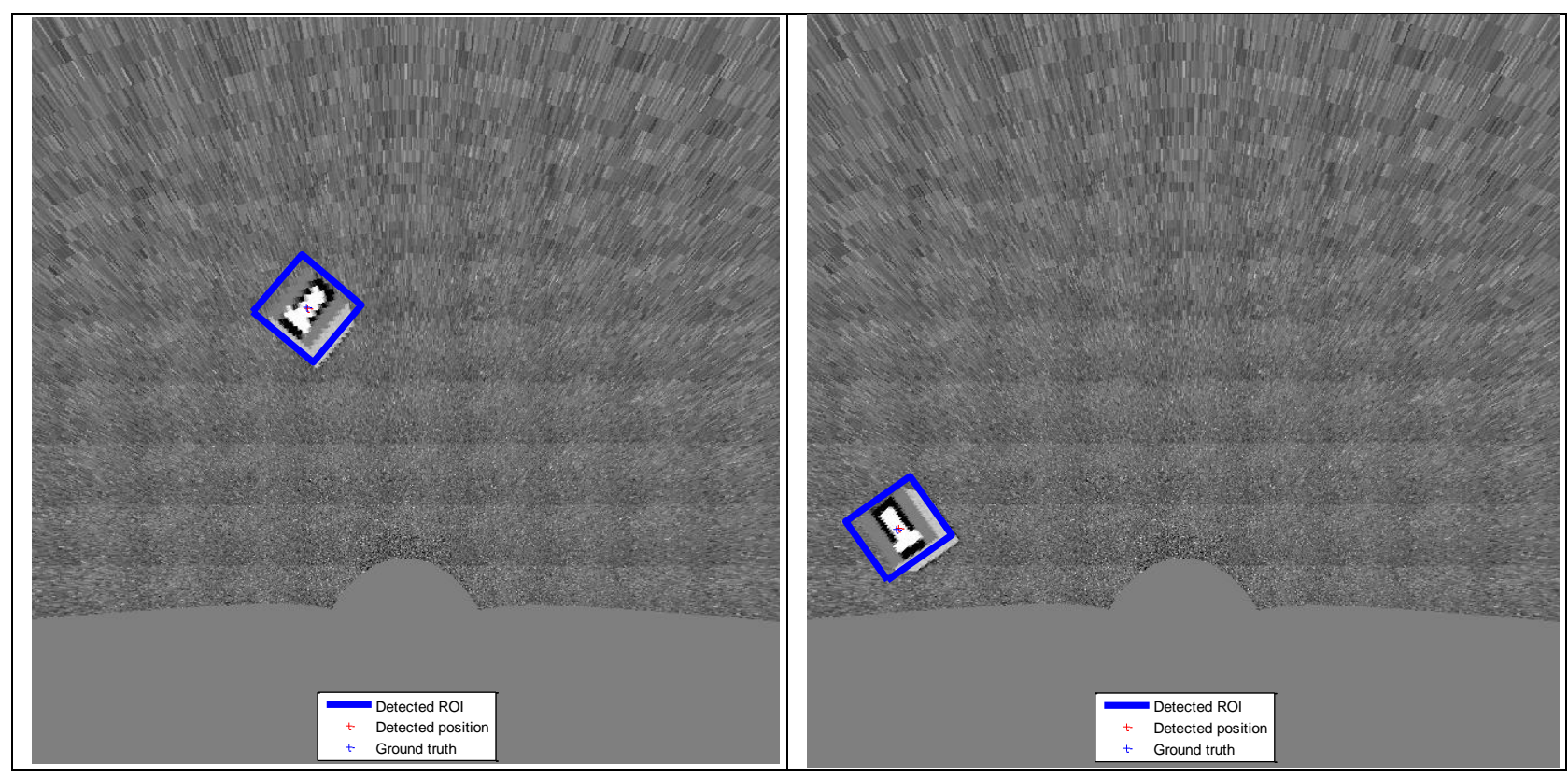

Figure 7. Example Detections

For this project, the requirements state that the detection system must be able to detect the pad at a 5 meter distance from the vehicle's charging system with a minimum precision of 5 centimetres. The vehicle's charging system is 1.5 meters behind the camera's point of view, so this constraint applies to a distance of $3.5 \mathrm{~m}$ from the camera.

\begin{tabular}{|c|c|c|c|c|c|}
\hline Methods & $\begin{array}{c}\text { Distance } \\
\text { (m) }\end{array}$ & $\begin{array}{l}\text { Average Error } \\
(\mathrm{cm})\end{array}$ & $\begin{array}{l}\text { Min Error } \\
(\mathrm{cm})\end{array}$ & $\begin{array}{l}\text { Max Error } \\
(\mathrm{cm})\end{array}$ & $\begin{array}{c}\text { Std dev } \\
\text { Error }\end{array}$ \\
\hline \multirow{6}{*}{ FFT-FFT } & 1.5 & 3.85 & 2.32 & 4.88 & 0.65 \\
\hline & 2.5 & 3.96 & 2.51 & 5.01 & 0.54 \\
\hline & 3.5 & 3.84 & 2.35 & 5.17 & 0.64 \\
\hline & 4.5 & 3.83 & 1.85 & 6.81 & 1.13 \\
\hline & 5.5 & 4.10 & 0.88 & 8.49 & 1.98 \\
\hline & 6.5 & 11.82 & 1.54 & 35.35 & 10.04 \\
\hline \multirow{4}{*}{ Seg-FFT } & 1.5 & 3.85 & 2.32 & 4.88 & 0.65 \\
\hline & 2.5 & 3.96 & 2.51 & 5.01 & 0.54 \\
\hline & 3.5 & 3.84 & 2.35 & 5.17 & 0.64 \\
\hline & 4.5 & 3.83 & 1.85 & 6.81 & 1.13 \\
\hline
\end{tabular}




\begin{tabular}{|c|c|c|c|c|c|}
\hline \multirow{4}{*}{} & 5.5 & 4.10 & 0.88 & 8.49 & 1.98 \\
\cline { 2 - 6 } & 6.5 & 10 & 1.54 & 35.24 & 9.05 \\
\hline \multirow{4}{*}{ Seg-CC } & 1.5 & 2.23 & 0.89 & 3.41 & 0.48 \\
\cline { 2 - 6 } & 2.5 & 2.30 & 1.27 & 3.34 & 0.45 \\
\cline { 2 - 6 } & 3.5 & 2.18 & 1.19 & 3.25 & 0.58 \\
\cline { 2 - 6 } & 4.5 & 2.16 & 0.30 & 4.23 & 1.05 \\
\cline { 2 - 6 } & 5.5 & 3.11 & 0.29 & 7.37 & 1.86 \\
\hline \multirow{5}{*}{ FFT-CC } & 6.5 & 5.60 & 0.71 & 17.98 & 5.18 \\
\cline { 2 - 6 } & 2.5 & 2.23 & 0.89 & 3.41 & 0.48 \\
\cline { 2 - 6 } & 3.5 & 2.30 & 1.27 & 3.34 & 0.45 \\
\cline { 2 - 6 } & 4.5 & 2.18 & 1.19 & 3.25 & 0.58 \\
\cline { 2 - 6 } & 5.5 & 2.16 & 0.30 & 4.23 & 1.05 \\
\cline { 2 - 6 } & 6.5 & 3.11 & 0.29 & 7.37 & 1.86 \\
\hline
\end{tabular}

Table 3. Comparison of the Errors Generated by the Different Methods

As can be seen from Table 3, the methods which give better results are those which use normalized cross-correlation for the localisation. Even though FFT methods are less accurate, the results comply on average with the requirements for distances below 5.5 meters.

The graph in Figure 8 shows the detected positions versus the ground truth in a top down view for each method. The circles represent the maximum error distance from the ground truth. 


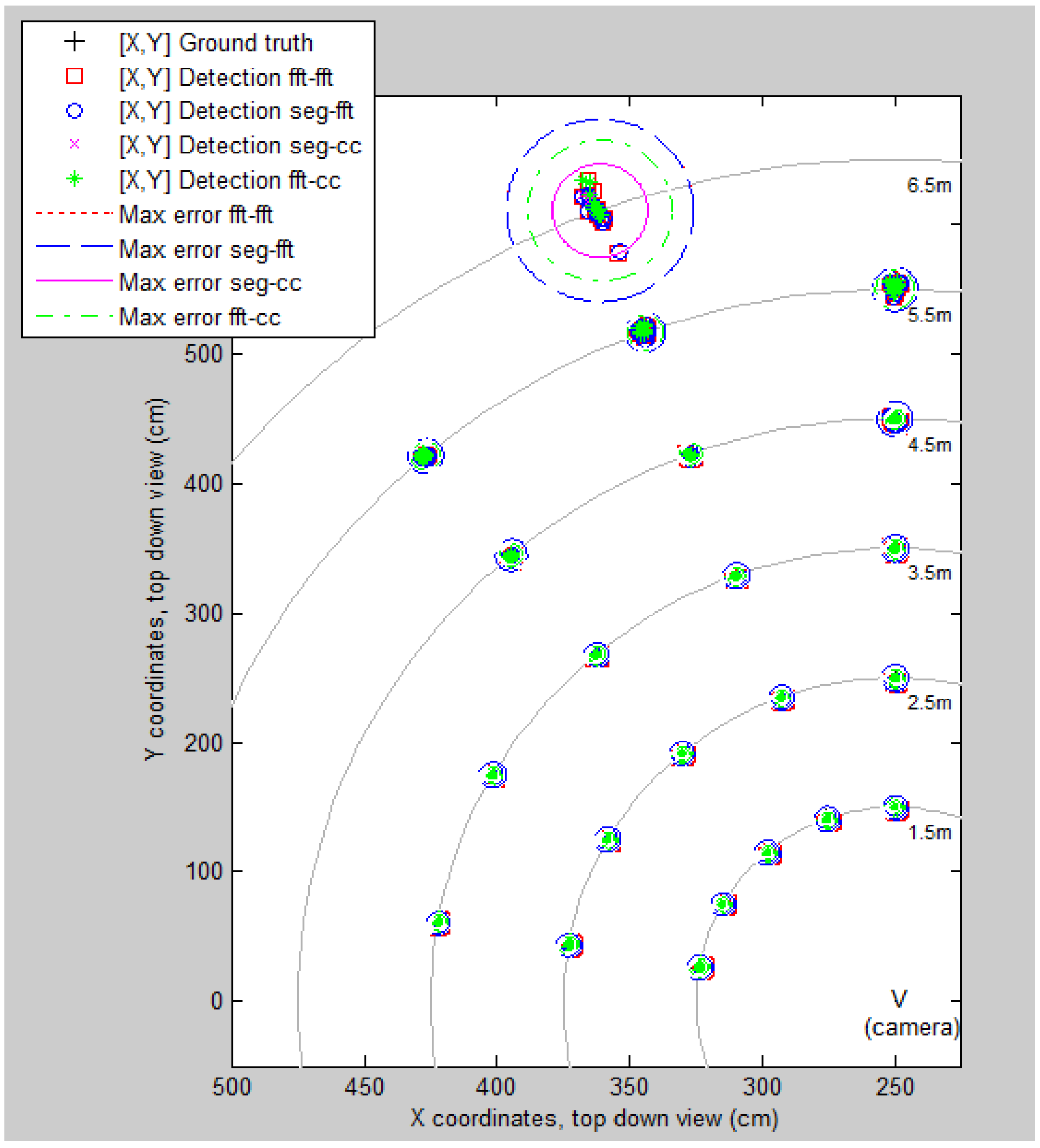

Figure 8. Detected Pad Position in Top Down View

Figure 9 shows the detected angles versus the ground truth in a top down view. 


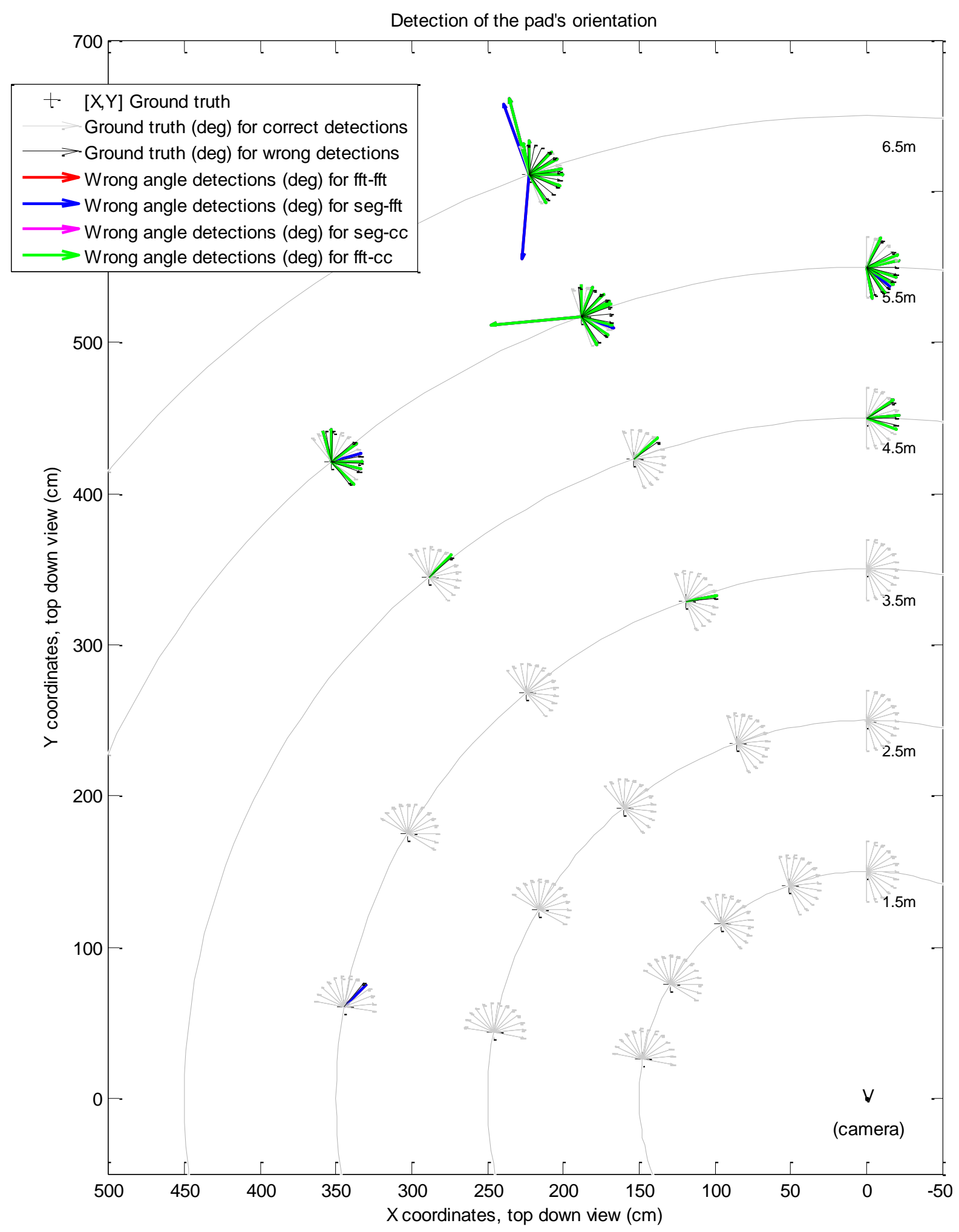

Figure 9. Detected Orientation 
The grey arrows show the pad's angles where all methods detected correctly the angle. The black arrows show the ground truth where not every method detected the angle correctly. Finally, the colour arrows show the detected angles per method when the detection is incorrect. In those cases, the arrow is orientated at the detected angle and its magnitude is proportional to the distance between that expected and then detected. Minor errors are visible at $4.5 \mathrm{~m}$ distance and further away they begin to be noticeable. At $6.5 \mathrm{~m}$ the angle detection is not reliable. Table 4 summarizes the reliability of accurate angle determination with distance from the template.

\begin{tabular}{|l|c|c|c|c|c|c|}
\hline Distance (m) & \multicolumn{2}{|l|}{$\%$ correct cases } & \multicolumn{2}{l|}{$\%$} \\
& \multicolumn{2}{|c|}{$\%$ cases precision $\pm 5^{\circ}$} & & \multicolumn{2}{l|}{ CC } \\
\hline & FFT & CC & FFT & CC & FFT & $100 \%$ \\
\hline $\mathbf{1 . 5}$ & $100 \%$ & $100 \%$ & $100 \%$ & $100 \%$ & $100 \%$ & $100 \%$ \\
\hline $\mathbf{2 . 5}$ & $100 \%$ & $100 \%$ & $100 \%$ & $100 \%$ & $100 \%$ & $100 \%$ \\
\hline $\mathbf{3 . 5}$ & $97 \%$ & $98 \%$ & $100 \%$ & $100 \%$ & $100 \%$ & $100 \%$ \\
\hline $\mathbf{4 . 5}$ & $95 \%$ & $87 \%$ & $100 \%$ & $100 \%$ & $100 \%$ & $97 \%$ \\
\hline $\mathbf{5 . 5}$ & $49 \%$ & $44 \%$ & $90 \%$ & $92 \%$ & $97 \%$ & $92 \%$ \\
\hline $\mathbf{6 . 5}$ & $23 \%$ & $31 \%$ & $62 \%$ & $77 \%$ & $77 \%$ & $\mathbf{1 0}^{\circ}$ \\
\hline
\end{tabular}

Table 4. Reliability of different methods with distance from pad

The execution time measured for the pre-localisation, the localisation and the sum of both is shown in Table 5 below.

\begin{tabular}{|l|l|l|l|l|}
\hline Time (ms) & FFT-FFT & Seg-FFT & Seg-CC & FFT-CC \\
\hline Pre-localisation & 3138 & 9 & 10 & 2863 \\
\hline Localisation & 1147 & 652 & 314 & 579 \\
\hline Total & 4285 & 661 & $\mathbf{3 2 4}$ & 3442 \\
\hline
\end{tabular}

Table 5. Algorithm execution times

\section{CONCLUSION}

In the conditions tested during this study the method developed, based on normalized two-dimensional correlation, works effectively. One of the strong points of the selected approach is the flexibility of using a two-step localisation method, with a coarse pre-localisation followed by a finer localisation stage. This allows the use of more complex approaches during the second step to obtain more precision.

Another relevant benefit of this study is the framework it provides in a Matlab environment to quickly test different approaches, different patterns and different images in a systematic manner. Should the requirements change, or new ideas arise, testing them and comparing the results will be easier and faster, helping in the decision-making process.

\section{REFERENCES}

[1] O. Hafez, K. Bhattacharya, "Optimal design of electric vehicle charging stations considering various energy resources", Renewable Energy, vol. 107, pp. 576-589, 2017 
[2] G. Yuqing, G. Yanning, X. Hang and F. Zhen, "Vision-Based Detection and Tracking of a Moving Target for a Smart Car," IEEE 3rd International Conference on Image, Vision and Computing (ICIVC), Chongqing, 2018, pp. 586-591.

[3] G. Bradski, A. Kaehler, Learning OpenCV: Computer Vision with the OpenCV Library. O'Reilly: Sebastopol, CA, 2008.

[4] H. Bay, A. Ess, T. Tuytelaars, L. Van Gool, SURF: "Speeded Up Robust Features", Computer Vision and Image Understanding (CVIU), Vol. 110, No. 3, pp. 346--359, 2008

[5] https://uk.mathworks.com/help/images/ref/corr2.html, 19/03/2018 Accessed $1^{\text {st }}$ March 2019

[6] H. Ye, G. Shang, L. Wang, M. Zheng, "A new method based on the Hough transform for quick line and circle detection", Biomedical Engineering and Informatics (BMEI) 2015 8th International Conference on, pp. 52-56, 2015.

[7] C. Guo, X. Li, L. Zhong, "A fast and accurate corner detector based on the Harris Algorithm 2009", Third International Symposium on Intelligent Information Technology Application, pp. 49-52, 2009

[8] M. Miura, S. Shuji, A. Shoichiro, I. Jumpei et al. High-Accuracy Image Matching Using Phase-Only Correlation and Its Application, SICE Annual Conference 2012, August 20-23, 2012, Akita University, Akita, Japan, page 307-312.

[9] H. Joo and J. W. Jeon, "Feature-point extraction based on an improved SIFT algorithm," 2017 17th International Conference on Control, Automation and Systems (ICCAS), Jeju, 2017, pp. 345-350.

[10] T. Dey and T. Deb, "Facial landmark detection using FAST Corner Detector of UGC-DDMC Face Database of Tripura tribes," Proceedings of the 2015 Third International Conference on Computer, Communication, Control and Information Technology (C3IT), Hooghly, 2015, pp. 1-4.

[11] M. Sridevi, N. Sankaranarayanan, A. Jyothish, A. Vats and M. Lalwani, "Automatic traffic sign recognition system using fast normalized cross correlation and parallel processing," 2017 International Conference on Intelligent Communication and Computational Techniques (ICCT), Jaipur, 2017, pp. 200-204. 